\title{
Connecting monotonic and oscillatory motions of the meniscus of a volatile polymer solution to the transport of polymer coils and deposit morphology
}

\author{
Mohammad Abo Jabal, ${ }^{\dagger}$ Ala Egbaria, ${ }^{\dagger}$ Anna Zigelman, ${ }^{\dagger}$ Uwe Thiele, ${ }^{\ddagger}$ and Ofer \\ Manor $^{*} \dagger$ \\ $\dagger$ Wolfson Department of Chemical Engineering, Technion - Israel Institute of Technology, \\ Haifa, Israel 32000 \\ $\ddagger$ Institut für Theoretische Physik, Westfälische Wilhelms-Universität Münster, Wilhelm \\ Klemm Str. 9, D-48149 Münster, Germany and Center of Nonlinear Science (CeNoS), \\ Westfälische Wilhelms Universität Münster, Corrensstr. 2, 48149 Münster, Germany \\ E-mail: manoro@technion.ac.il
}

\begin{abstract}
We study the connection between the polymer deposition patterns to appear following the evaporation of a solution of poly-methyl-methacrylate (PMMA) in toluene, the transport of the polymer coils in the solution, and the motion of the meniscus of the solution. Different deposition patterns are observed when varying the molecular mass and initial concentration of the solute and temperature and are systematically presented in the form of morphological phase diagrams. The modi of deposition and meniscus motion are correlated. They vary with the ratio between the evaporation-driven convective
\end{abstract}


flux and diffusive flux of the polymer coils in the solution. In the case of a diffusiondominated solute transport, the solution monotonically dewets the solid substrate by evaporation, supporting continuous contact line motion and continuous polymer deposition. However, a convection-dominated transport results in an oscillatory ratcheting dewetting-wetting motion of the contact line with more pronounced dewetting phases. The deposition process is then periodic and produces a stripe pattern. The oscillatory motion of the meniscus differs from the well documented stick-slip motion of the meniscus, observed as well, and is attributed to the opposing influences of evaporation and Marangoni stresses, which alternately dominate the deposition process.

\section{Introduction}

Solutions and suspensions, comprising a volatile solvent and a polymer, macromolecules like collagen or DNA, nanoparticles, or carbon nanotubes were found to produce intriguing oneand two-dimensional deposition patterns at receding three-phase contact lines on solid substrate where the motion is induced by solvent evaporation. Examples include deposits that consist of one or multiple rings ("coffee ring effect"), 12 branched patterns resulting from fingering instabilities of the receding contact line, ${ }^{3 / 4}$ and regular periodic stripe patterns. $\frac{[5] 6}{56}$ Moreover, pattern deposition in a restricted geometry has been introduced as a simple preparation route for the fabrication of microscopic structures of high fidelity and regularity. ${ }^{78}$ The deposited patterns find applications in sensing, ${ }^{9}[14$ data storage,, $15[16$ and photonic band gap materials ${ }^{17 / 18}$ as well as in the fabrication of nanostructured templates ${ }^{19 \mid 20}$ and of ordered porous materials. 2124

The morphology of the deposits is a consequence of the concurrent interplay of several mechanisms, including the flow in the liquid which is generated by the evaporation of the solvent, Marangoni-force and capillarity induced flows, 2531 etc. Furthermore, the evaporation of a macroscopic droplet or an unbounded film into an open vapor atmosphere usually yields rather irregular patterns of rugged rings or lines. $\frac{32-36}{36}$ However, restraining the liquid, 
using physical confinement, is known to yield well organized deposits. $\frac{35}{35}$ Examples for such

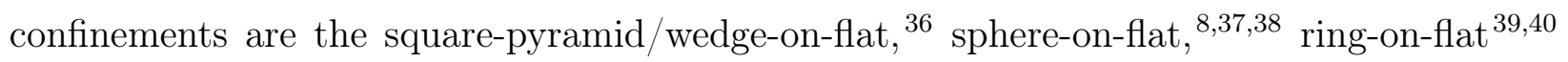
and cylinder-on-flat ${ }^{41}$ geometries as well as two paralle ${ }^{[2942143}$ or tilted plates, crossed cylinders, $\frac{44}{4}$ and capillary tubes. ${ }^{45}$ Recent reviews of different aspects of these systems are given elsewhere. .30135146

There are few studies that analyze the properties of regular patterns, which are deposited in confined geometries. Yabu et al. ${ }^{47}$ employed a computer-controlled apparatus comprising two precisely manipulated sliding parallel glass plates. A polymer solution (polystyrene dissolved in chloroform) was continuously fed into the gap $(200 \mu \mathrm{m})$ between the two plates while the upper plate slid over the lower one. The polymer solution formed a meniscus that moved along with the edge of the upper plate. Various types of polymer patterns were left behind the meniscus on the bottom plate following the evaporation of the solvent. Increasing the polymer concentration, three different modi of contact line motion were found, namely, transversally invariant (i.e., along the contact line) continuous receding motion, transversally invariant stick-slip motion, and continuous receding of a contact line with fingering instability. These modi of contact line motion gave rise to three respective types of micrometer-scale patterns, namely, rhombic dot patterns, stripe patterns, and ladderlike patterns of orthogonal sets of lines. Moreover, changes in the sliding speed of the plate influenced the morphology of the patterns. In another work, Hong et al. $\frac{48}{\text { evaporated }}$ a polymer solution (polystyrene dissolved in toluene) in a capillary structure formed by a sphere-on-flat geometry. They found that the variation in the molecular weight, $M_{w}$, of polymers can lead to a pronounced change in the morphology of the deposit. At low $\mathrm{M}_{\mathrm{w}}$ values, the evaporative dewetting process left behind randomly distributed dots. At intermediate $\mathrm{M}_{\mathrm{w}}$ values, concentric rings were formed by a cyclic deposition-recession process, while high $M_{w}$ produced concentric rings, rings with fingers, and punch-hole-like structures. In addition, $\mathrm{Xu}$. et al.,$^{8}$ investigated the effect of polymer concentration on the morphology of the concentric rings pattern obtained by evaporating a solution of poly[2-methoxy-5-(2- 
ethylhexyloxy)-1,4-phenylenevinylene] in toluene in a sphere-on-flat geometry. They found that the distance between adjacent rings and their thickness increased with increasing the concentration of the polymer solution.

In the present work, we investigate the physical processes that are responsible for qualitative changes in the pattern deposition of a polymer from a volatile solution, and discuss the corresponding changes in contact line motion. The paper is structured as follows. In "Experimental Methods" we describe our experimental system and procedures of measurement and analysis. In "Experimental Results" we present different deposition patterns and morphologies of the polymer and corresponding morphological phase diagrams. We discuss in detail the variations in the morphology of the deposits and connect them to changes in the modus of contact line motion. In "Discussion" we highlight the interplay of the diffusive and advective transport of the polymer chains in the volatile solution and its contribution to the bifurcations occurring in the deposition process, and in "Conclusion" we conclude our findings.

\section{Experimental Methods}

As a model experimental system we place a solution of poly-methyl methacrylate (PMMA, 9011-14-7, Aldrich) in toluene $(99.8 \%, 108-88-3$, Aldrich) in a micro-chamber of rectangular geometry as illustrated in Fig. 1. The chamber is composed of an upper glass cover (micro-

scope slide, Marienfeld), a U-shaped spacer made of polyamide (Kapton, DuPont), and a substrate of silicon oxide (atop a silicon wafer $<100>, 550 \mu \mathrm{m}$ thickness, Silicon Materials). We employ polymers chains of average molecular masses of $\mathrm{M}_{\mathrm{w}}=10,000,50,000,100,000$, and $350,000 \mathrm{Da}$ at initial solute concentrations of $C=0.5,1,3,5,7,10,13$, and $15 \mathrm{mg} / \mathrm{ml}$. All initial concentrations are below the overlap concentration, i.e., steric and frictional interactions of neighbouring polymer chains are negligible (dilute solutions). We further employ a chamber with an inner width of $H=75 \mu \mathrm{m}$. 

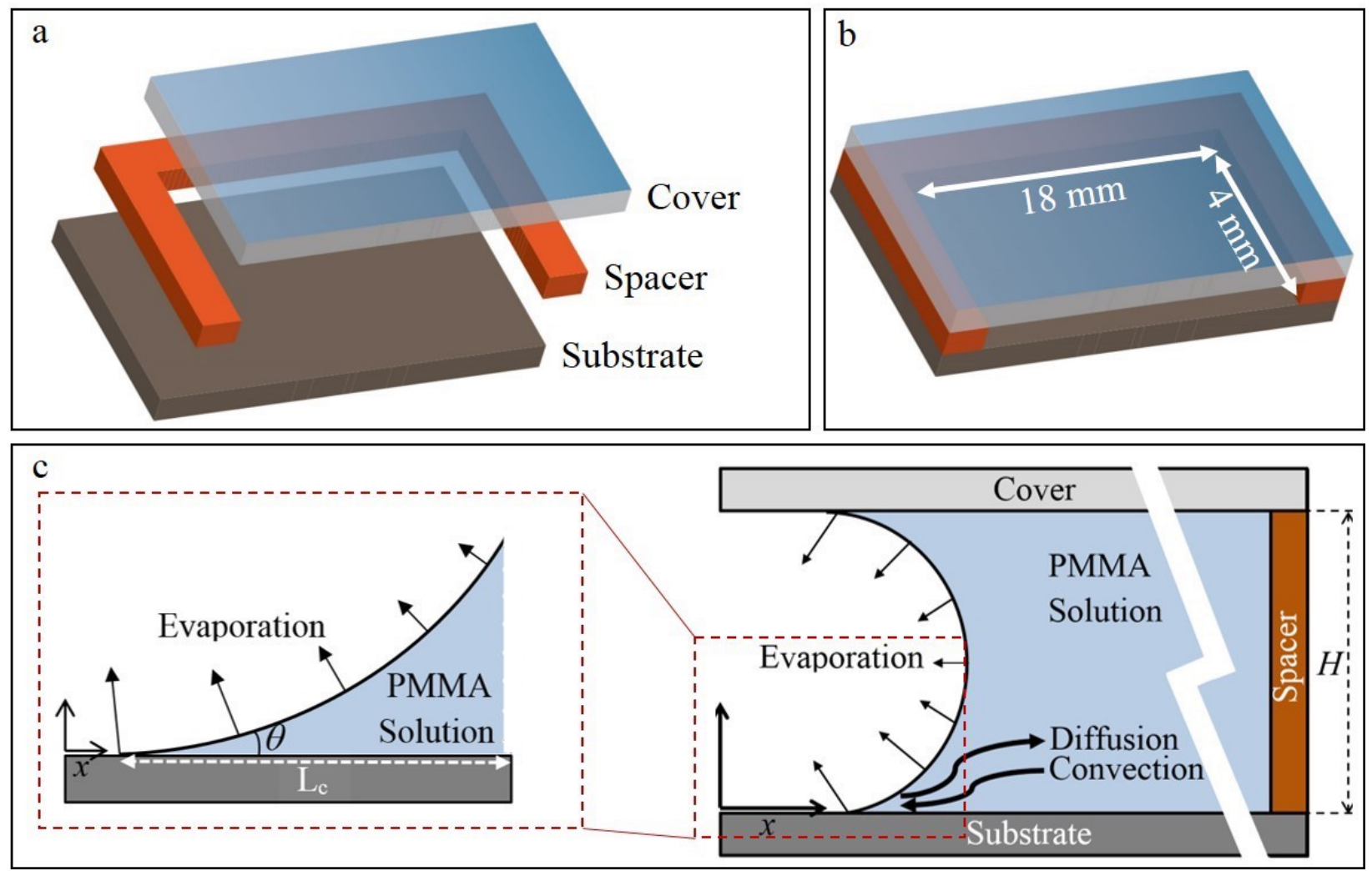

Figure 1: (a) A sketch of the different parts of the micro-chamber shown in (b) and (c) an illustration of the cross-sectional profile of the evaporating solution in the micro-chamber, where $\theta$ and $H$ are the contact angles of the solution with the solid substrate and the inner width of the chamber, respectively.

Prior to each experiment we clean the three parts of the chamber by dipping them in a hot bath of acetone, ethanol, isopropyl alcohol, followed by drying them in a stream of air, rinsing in Milli-Q water $(18 \mathrm{M} \Omega \mathrm{cm})$, and finally drying again. The substrates are then treated for 5 min using a plasma cleaner (PDC-001-HP (115V), Harrick Plasma).

Each surface is characterised by measuring the apparent receding and advancing contact angles using a goniometer (Data Physics; OCA 15Pro). The receding and advancing contact angles measured for toluene on silicon oxide are $10^{\circ} \pm 2^{\circ}$ and $12^{\circ} \pm 2^{\circ}$, respectively, while for toluene on a glass cover these angles are measured to be $14^{\circ} \pm 2^{\circ}$ and $19^{\circ} \pm 2^{\circ}$, respectively. The micro-chamber is then placed on a heating plate inside a laminar fume hood (Optimal 12, Stérile Laminaire). As the liquid evaporates, the three-phase contact line between the toluene, its vapor, and the silicon oxide substrate moves (on average) toward the interior of 
the chamber. The motion of the contact line, the deposition process, and the morphology of the polymer deposits are recorded by a monochromatic camera (Q Imaging, Nikon) mounted on an upright Nikon eclipse optical microscope. Moreover, our measurements of the dynamic position of the contact line further give the temporal rate of evaporation of the meniscus in the chamber versus the distance between the contact line and the open end of the chamber.

\section{Experimental Results}

We distinguish between three characteristic regimes of contact line motion (see movie SI1 in Supplemental Information). In the first regime, we observe that the contact line moves continuously and monotonically while its distance from the open end of the chamber increases due to evaporation. This happens when employing polymers of low molecular mass, namely, $M_{w}=10,000 \mathrm{Da}$ and $M_{w}=50,000 \mathrm{Da}$, at low temperatures and polymer concentrations. The position of the contact line versus time and the deposit that it leaves behind are visualized in the sequence of video frames shown in Fig. $2\left(b_{1}-b_{3}\right)$. The corresponding dependence of the position of the contact line on time is shown in Fig. 3 as a black solid line. The process results in a continuous deposition of either uniform coatings or of continuous structures in the form of polygonal networks; both are shown in Fig. 4 (a-c).

The second regime appears when employing the polymer of our largest molecular mass $\left(M_{w}=350,000 \mathrm{Da}\right)$. Here we observe a different type of contact line dynamics as it undergoes a non-monotonous oscillatory wetting-dewetting motion cycles that are correlated to the deposition of lines. A sequence of video frames visualizing the displacement of the contact line during two cycles and the resulting deposit is shown in Fig. 2 $\left(a_{1}-a_{6}\right)$, while the red solid curve in Fig. 3 gives the contact line position over time and clearly indicates a nearly periodic change of the contact line speed. Specifically, each cycle is composed of three stages. Initially, the three-phase contact line recedes for a finite time, i.e., the solution dewets the substrate due to solvent evaporation. This stage is then followed by the motion of the contact line into 


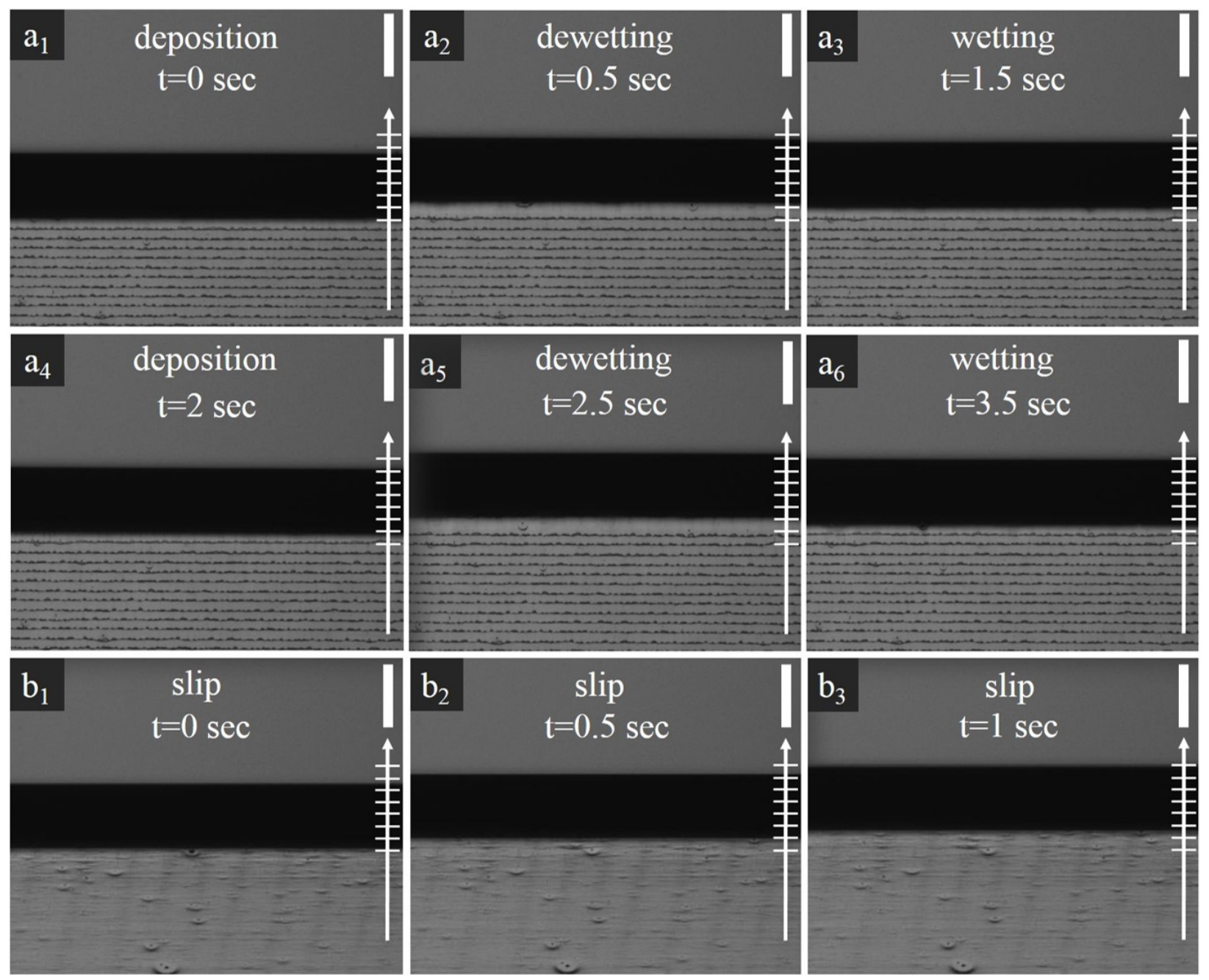

Figure 2: Three sequences of video frames (top views) that show time evolutions of the contact line region (meniscus position) and the resulting deposition patterns (lower part of each image) in the course of the evaporative dewetting in the micro-chamber. The large black rectangle in the middle of each image is the curved meniscus. The homogeneously gray parts above it correspond to the part of the chamber which contains the solution. The polymer deposit in the lower part of each frame is nearly homogeneous in the bottom row $\left(b_{1}-b_{3}\right)$, and shows a regular stripe pattern in the top and middle rows $\left(a_{1}-a_{6}\right)$. The corresponding dependencies of the contact line position on time are given by the black and red solid lines in Fig. 3, respectively. The only varied parameter is the molecular mass of the polymer that is $M_{w}=350,000 \mathrm{Da}$ in $\left(a_{1}-a_{6}\right)$ and $M_{w}=50,000 \mathrm{Da}$ in $\left(b_{1}-b_{3}\right)$. The open end of the micro-chamber is located at the bottom of each image, the white scale bar is $50 \mu \mathrm{m}$, and the separation between the ticks on the white arrow that indicates the direction of overall contact line motion is $10 \mu \mathrm{m}$. The inner width of the chamber is $H=75 \mu \mathrm{m}$, the temperature is $T=25^{\circ} \mathrm{C}$, and the initial solution concentration is $C=3 \mathrm{mg} / \mathrm{ml}$.

the opposite direction for a finite time, i.e., the solution wets the substrate again. During these two steps the polymer accumulates close to the contact line. Next, the solution recedes 
again (dewets the solid) while, leaving a stripe of polymer behind. Moreover, the distance that the contact line traverses during the dewetting stage is greater than during the wetting stage. The combined displacements of the contact line in each cycle result in an overall dewetting motion, which is well visualized in Fig. 3. We refer to this modus of contact line motion as an "oscillatory wetting-dewetting motion". The corresponding deposits correspond to discontinuous stripes, solid continuous stripes, and continuous stripes with moderate or extreme fingering instabilities, as shown in Fig. 4(f-i)

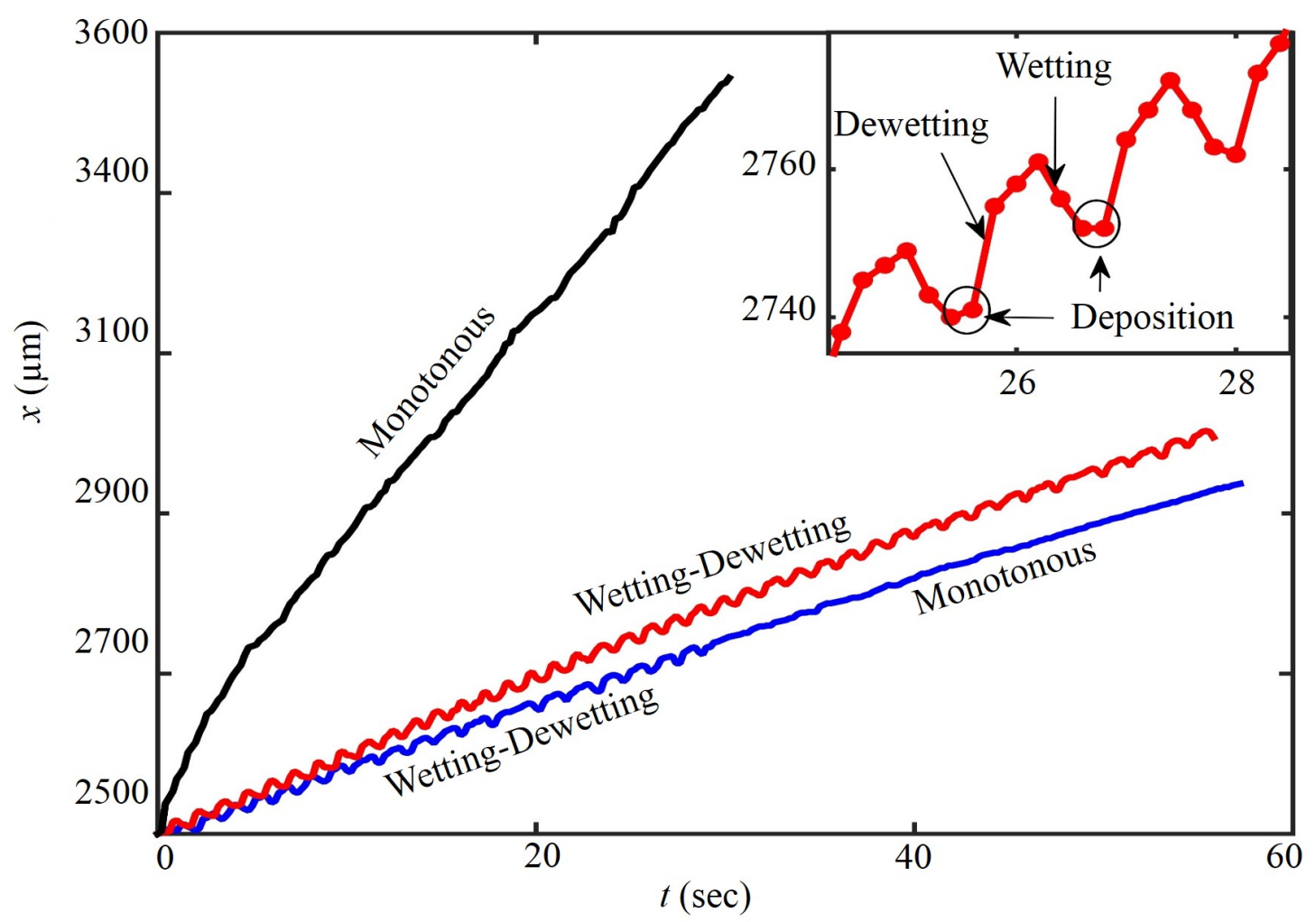

Figure 3: Time $(t)$ variations of the contact line position $(x)$ for the different modi of contact line motion in a micro-chamber, given by a monotonous motion at a molecular mass of $M_{w}=50,000 \mathrm{Da}$ (black line), an oscillatory wetting-dewetting motion at $M_{w}=350,000 \mathrm{Da}$ (red line), and a transition from oscillatory to monotonous mottions at $M_{w}=100,000 \mathrm{Da}$ (blue line) at a temperature of $T=25^{\circ} \mathrm{C}$, and with a solution concentration of $C=3$ $\mathrm{mg} / \mathrm{ml}$, where the open end of the chamber is at $x=0$. The inset gives an enlarged view of a few periods of the oscillatory wetting-dewetting motion.

In the case of moderate values of the molecular mass, namely, $M_{w}=50,000$ Da (at relatively high temperatures and polymer concentration in the solution) and $M_{w}=100,000$ Da, 
we initially observe an oscillatory wetting-dewetting motion of the contact line that later transforms into a monotonic motion when the meniscus has receded deeper into the chamber, shown in Fig. 3 as a blue solid line. The patterns which correspond to this regime are continuous and discontinuous stripes that are then replaced by a uniform polymer film as shown in Fig. 4(d,e).

The state of deposition was generated by choosing specific combinations of the control variables, namely, temperature, polymer concentration, and molecular mass. The different deposition patterns are displayed in Fig. 4. The letters "a"- "i" that indicate the individual states of deposition correspond to regions in the morphological phase diagrams in Fig. 5 . In Fig. 4 (a) we show a non-uniform coating that occurs in the regions denoted by "a" in the panels in Fig. 5. Essentially, this region is characterized by low initial polymer concentrations (below $0.5 \mathrm{mg} / \mathrm{ml})$. At low molecular mass $\left(M_{w}=10,000,50,000 \mathrm{Da}\right)$, low temperature, and low concentration, the deposits take the shape of an continuous polygonal network,, 49 as shown in Fig. 4(b) and marked by "b" in Fig. 5. Increasing the temperature and concentration results in the deposition of a uniform film (shown in Fig. 4(c) and marked by "c" in Fig. 5). Deposits that show transients from discontinuous stripes to uniform deposition are shown in Fig. $4(\mathrm{~d})$ and result in the regions denoted by "d" in Fig. 5 . At larger molecular mass $\left(M_{w}=100,000 \mathrm{Da}\right)$, we additionally observe a transition from the transient patterns marked by "d" in Fig. 5 to the deposition modus shown in Fig. 4(e) and marked by "e" in the middle row in Fig. 5. The deposits change from continuous stripes to a dendrite-like pattern. ${ }^{2}$ Further increasing the temperature and the initial concentration brings us to region "f" in Fig. 5 (see pattern in Fig. $4(\mathrm{f})$ ) where discontinuous stripe patterns are found. Finally, a further increase in the molecular mass (reaching $M_{w}=350,000 \mathrm{Da}$ ) at low temperature and concentration gives again the patterns in "d" in Fig. 5. Increasing the initial concentration, but keeping the temperature fixed, gives the deposits "e" in Fig. 5. However, increasing the temperature for concentrations above $1 \mathrm{mg} / \mathrm{ml}$, we observe an additional type of pattern, namely, a regular continuous stripe pattern (Fig. 4 (g), marked "g" in the lower right panel 

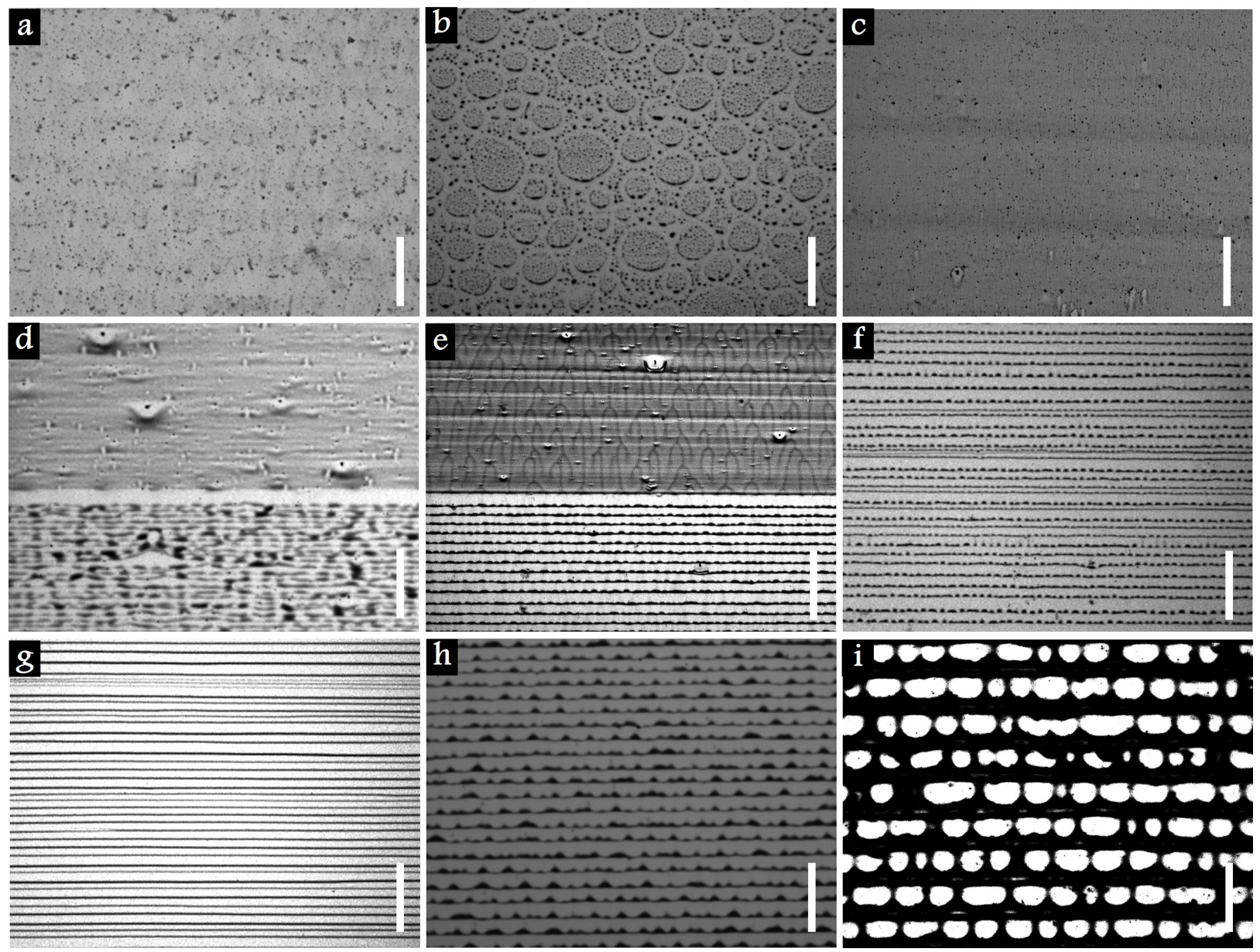

Figure 4: Typical deposition patterns, where we show in (a) a non-uniform coating $\left(M_{w}=\right.$ $\left.10,000 \mathrm{Da}, T=25^{\circ} \mathrm{C}, C=0.5 \mathrm{mg} / \mathrm{ml}\right)$, in (b) a polygonal network pattern $\left(M_{w}=10,000\right.$ Da, $\left.T=25^{\circ} \mathrm{C}, C=3 \mathrm{mg} / \mathrm{ml}\right)$, in (c) a uniform coating $\left(M_{w}=50,000 \mathrm{Da}, T=25^{\circ} \mathrm{C}\right.$, $C=3 \mathrm{mg} / \mathrm{ml})$, in (d) discontinuous stripes followed by uniform coating $\left(M_{w}=100,000 \mathrm{Da}\right.$, $T=25^{\circ} \mathrm{C}, C=3 \mathrm{mg} / \mathrm{ml}$ ), in (e) continuous stripes followed by a dendrite-like pattern in an otherwise uniform coating $\left(M_{w}=100,000 \mathrm{Da}, T=25^{\circ} \mathrm{C}, C=7 \mathrm{mg} / \mathrm{ml}\right)$, in (f) discontinuous stripes $\left(M_{w}=100,000 \mathrm{Da}, T=50^{\circ} \mathrm{C}, C=7 \mathrm{mg} / \mathrm{ml}\right)$, in (g) continuous stripes, i.e., a typical "stripe pattern," ( $M_{w}=350,000 \mathrm{Da}, T=25^{\circ} \mathrm{C}, C=3 \mathrm{mg} / \mathrm{ml}$ ), in (h) continuous stripes with moderate fingering instability $\left(M_{w}=350,000 \mathrm{Da}, T=35^{\circ} \mathrm{C}, C=3 \mathrm{mg} / \mathrm{ml}\right)$, and in (i) a more extreme fingering instability termed "punch-hole-like" $\left(M_{w}=350,000 \mathrm{Da}, T=65^{\circ} \mathrm{C}\right.$, $C=7 \mathrm{mg} / \mathrm{ml}$ ). The open end of the micro-chamber is located at the bottom of each image and the white scale bar is $100 \mu \mathrm{m}$.

of Fig. 5), which becomes punctured by fingering instability (Fig. 4(h), marked "h" in the lower right panel of Fig. 5, 50 and the "punch-hole-like" pattern (Fig. 4(i)) marked by "i". 37 

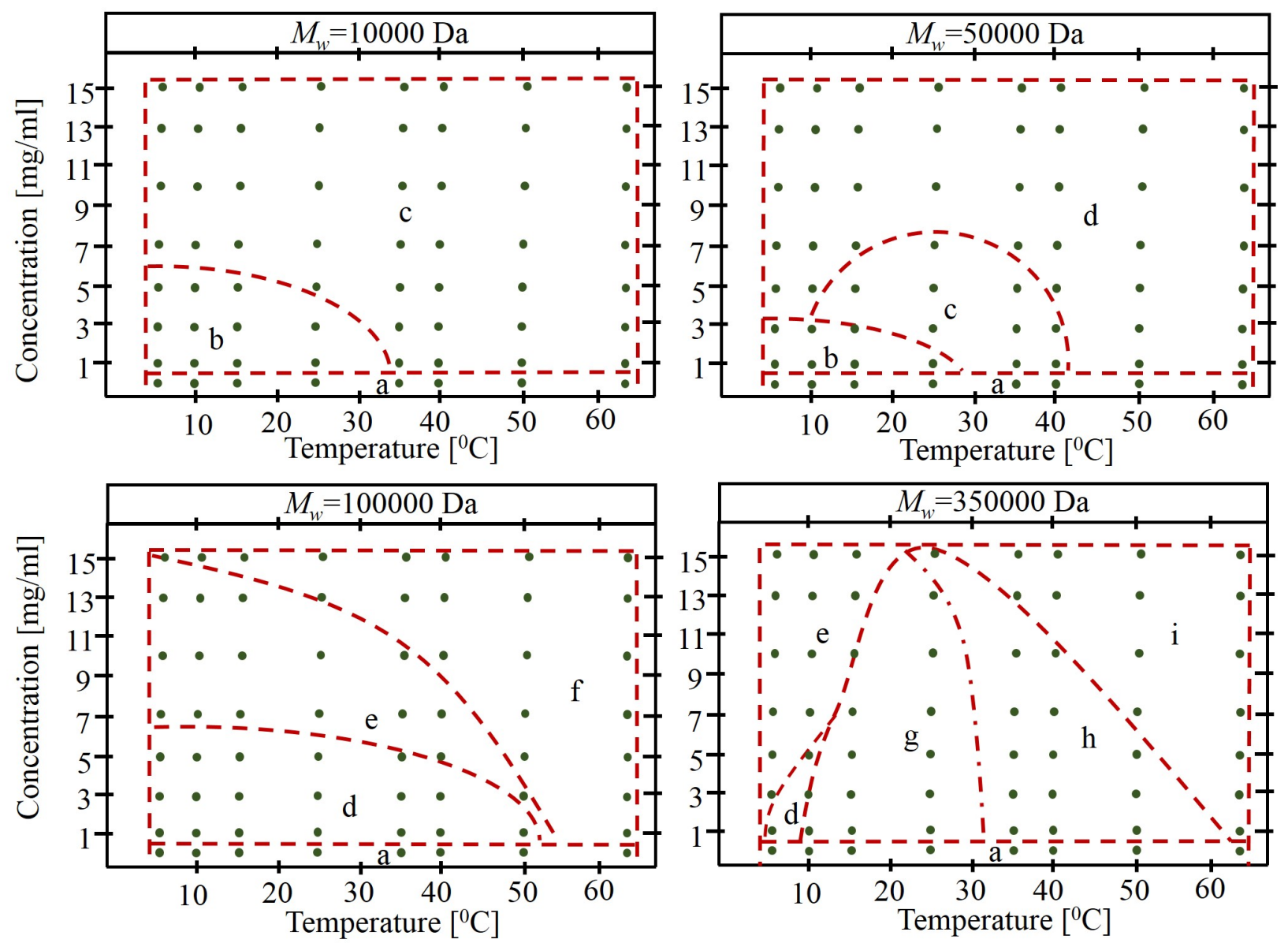

Figure 5: Morphological phase diagrams: Temperature and concentration variations of the deposition patterns "a" to "i" in Fig. 4 for different polymer molecular masses, $M_{w}$, where the approximate boundaries between different deposition modi are marked as dashed red lines and each green dot represents an experimentally obtained result.

\section{Discussion}

\section{Modi of deposition}

Next we discuss the physical mechanisms which determine the motion of the contact line and the corresponding modi of continuous or patterned deposition of the polymer from the volatile solution. The dynamics of the contact line appears to be connected to the ratio between the rates of solvent evaporation-driven convection of the solution and of polymer diffusion 
within the solvent. This is captured by the Péclet number, Pe, for polymer transport,

$$
\mathrm{Pe} \equiv \frac{L_{c} \varepsilon_{\mathrm{ev}}}{D_{l} \theta} .
$$

Here, $D_{l}, L_{c}, \theta$, and $\varepsilon_{\mathrm{ev}}$ are the diffusion coefficient of the solute, the characteristic length scale of the meniscus (shown in Fig. 11 ), the contact angle of the liquid with the substrate, and the evaporation rate per unit area, respectively; the velocity of the liquid along the solid is approximately $\varepsilon_{\mathrm{ev}} / \theta$. Further, the diffusion coefficient is a function of the molecular mass of the polymer chains, where according to de Gennes ${ }^{52} D_{l}=K M_{w}^{-a}$ and $a \simeq 2$ is the corresponding power for a diluted polymer solution (below the overlap concentration). Hence,

$$
\mathrm{Pe} \equiv \frac{\varepsilon_{\mathrm{ev}} L_{c} M_{w}^{a}}{K \theta} .
$$

The measured evaporation rate is approximately $\varepsilon_{\mathrm{ev}} \approx 10^{-7} \mathrm{~m} / \mathrm{s}$ (see the experimental section) at a temperature and an initial concentration of $T=25^{\circ} \mathrm{C}$ and $C=3 \mathrm{mg} / \mathrm{ml}$, respectively. The contact angle is estimated as the measured receding contact angle, $\theta \approx$ $0.17 \mathrm{rad}\left(\simeq 10^{\circ}\right)$, and the characteristic length is taken to be the approximate radius of the meniscus, namely, $L_{c} \approx H / 2 \theta$.

With these values, we show in Fig. 6 that the Péclet number increases by nearly two orders of magnitude across the experimental change of the molecular mass of the polymer from $M_{w}=10,000 \mathrm{Da}$ to $M_{w}=350,000 \mathrm{Da}$. Thus, when $M_{w}$ is small, the diffusion of polymer in the solvent may equilibrate the distribution of polymer within the meniscus faster than the evaporation of solvent convects the polymer toward the contact line. As a result, the concentration within the meniscus should be nearly uniform. The deposition of the polymer is then continuous and nearly uniform, as shown in Fig 4(c). The upper left panel in Fig. [5 shows that this is the prevalent modus of deposition for the lowest investigated molecular mass $\left(M_{w}=10,000 \mathrm{Da}\right)$ if the concentration and/or temperature are sufficiently high. 
The distinction between the non-uniform coating in Fig 4(a) and the polygonal network pattern in Fig 4(b) is likely to result from different dewetting mechanisms of an ultrathin moist or a mushy polymer layer that is left behind the receding contact line. This phenomenon was observed previously in the case of evaporatively dewetting nanoparticle suspensions (see Ref. ${ }^{\sqrt{4}}$ ) and is a reminiscent of the result of a homogeneous thin film of evaporating polymer solution. The nearly continuous patterns in Figs. 4(a,b) indicate that the final shape of the deposit is independent of the motion direction of the meniscus. As for evaporating aqueous solutions of collagen, studied by Mertig et al. ${ }^{53}$, Thiele et al. ${ }^{54}$, a film with small holes (as in Fig. 4(a)) or a polygonal network (as in Fig 4(b)) may result when spinodal dewetting or dewetting via nucleation dominate, respectively. For further detail see the discussions about the two dewetting modes for nonvolatile films ${ }^{55156}$ and about polygonal network structures, resulting from the dewetting of various volatile and nonvolatile thin films, given elsewhere ${ }^{5760}$.

At large molecular mass of the polymer the Péclet number is large, so that the diffusion of the polymer in the solvent is slow when compared to convective flows resulting from evaporation. Hence, the diffusion of polymer coils is not able to equilibrate the polymer concentration within the meniscus. The evaporation of the solvent results in the build up of high polymer concentrations close to the contact line. This results in the deposition of different types of pronounced non-continuous patterns, as shown in Figs. 4 (f) to (i), and is accompanied by oscillatory wetting-dewetting or stick-slip motions of the receding contact line, shown in Figs. 3.

We now bring our non-continuous deposits into the context of results obtained by Fraštia et al., $\stackrel{276162}{2}$ which modeled related systems by employing thin-film (long-wave) equations for the time evolution of the film height and the local amount of solute. ${ }^{6364}$ They defined a reciprocal Péclet number, $\mathrm{Pe}^{-1}$, as the ratio between the time scales of the wettability-driven dewetting and solute diffusion. In particular, they studied the case of a straight evaporatively and convectively receding front of a dewetting (infinite-length) film of nanoparticle 
suspension and showed that a viscosity that strongly increases with solute concentration is a mechanism for structured deposition. In the regime of line deposition, they described a periodic pinning and depinning dynamics of a receding liquid front to result from a periodic transition between convection-dominated and evaporation-dominated regimes of the front motion.

Fraštia et al. $\stackrel{27}{[1}$ further determined the connection between the properties of line patterns, such as length amplitude and period, and various physical parameters. They mainly studied the contributions to the deposition process from evaporation rate and initial solute concentration and described various transitions from uniform to line deposition. Their analysis corresponds to the presently described transitions from the morphology of the deposits given in Figs. 4(a) to (c) to the ones in Figs. 4(f) to (i). Furthermore, parts of the phase diagrams in Fig. 5 qualitatively agree with the lower left part of Fig. 9 in Ref. .27 Here we take into account that an increase in the evaporation number in the work by Fraštia et al. correlates to an increase in the temperature employed as a control parameter. Fraštia et al. also found that line deposition ceases again at large values of the evaporation number and initial concentrations, which are beyond the parameter values studied here.

The influence of solute diffusion was specifically studied in Section 3.5 of Ref. ${ }^{27}$ There it was demonstrated that large Péclet numbers (range $10^{4}-10^{5}$ ) support the deposition of stripes. However, reducing the Péclet number towards $10^{3}$ suppresses stripe deposition as then the receding dewetting front is not able to accumulate the solute, resulting in a uniform coating. This corresponds to the general tendency that we observe when moving from the lower right panel of Fig. 5 to the upper left one, i.e., decreasing $M_{w}$ and therefore the Péclet number. This agreement remains valid even though our present definition of the Péclet number slightly differs in the two studies. However, in both cases the Péclet number varies across the transition range by about two orders of magnitude (Fig. 6(b)).

We now discuss the transition states shown in Figs. 4(d,e) that consist of two clearly distinguished regions with different patterns. They occur at intermediate Péclet numbers or, 

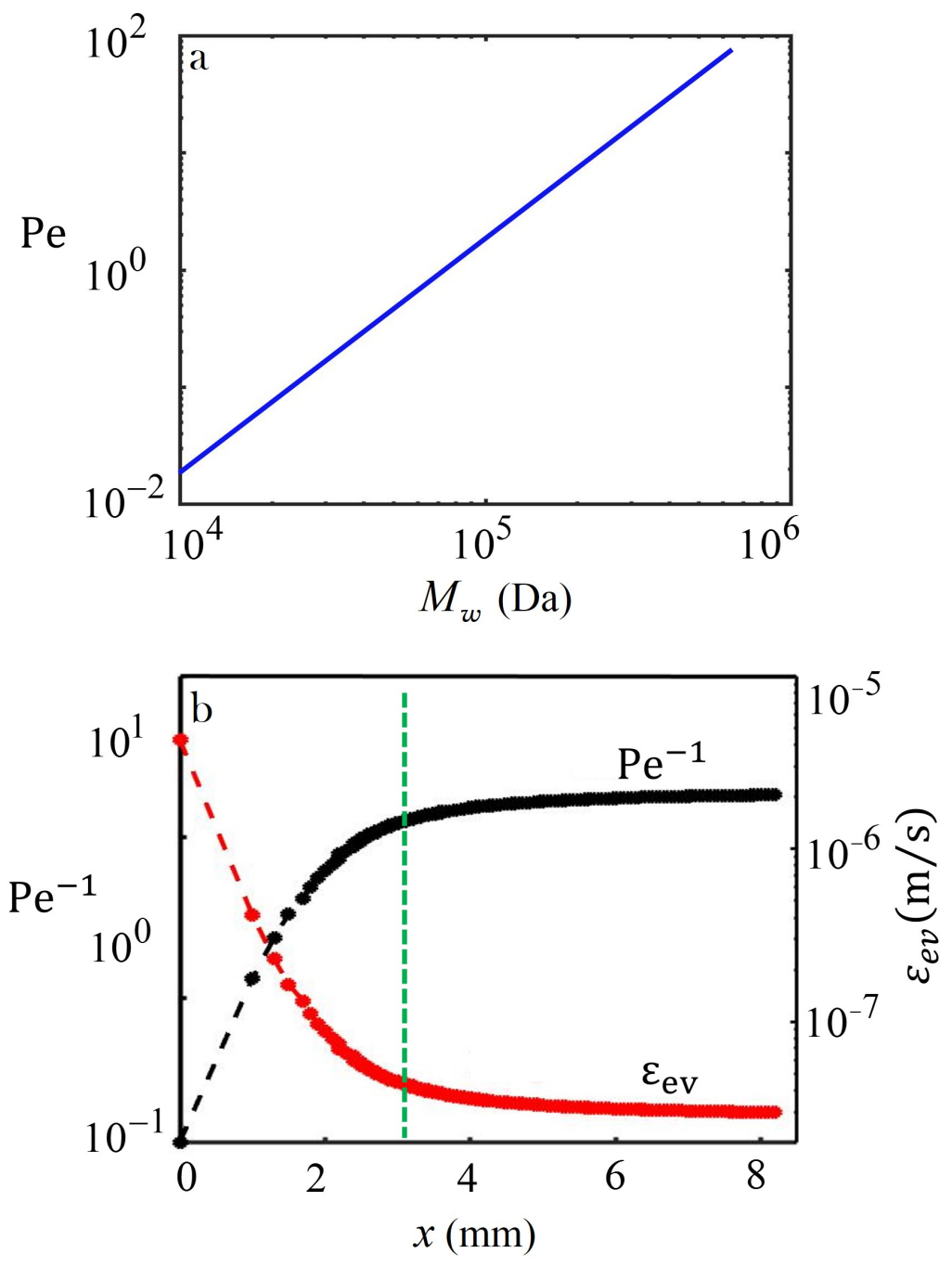

Figure 6: (a) Molecular mass, $M_{w}$, variations of the Péclet number, Pe, where we assumed $K \simeq 10^{-1} \mathrm{~m}^{2} \mathrm{gr}^{2} / \mathrm{s} \mathrm{mol}^{2}$ based on the de-Gennes correlation for a given diffusion coefficient of PMMA in Toluene and data given elswhere, ${ }^{65} T=25^{\circ} \mathrm{C}, C=3 \mathrm{mg} / \mathrm{ml}, \theta \simeq 0.17 \mathrm{rad}$, $H=100 \mu \mathrm{m}$, and $\varepsilon_{\mathrm{ev}}=10^{-7} \mathrm{~m} / \mathrm{s}$. (b) Separation (contact line to open end of chamber, $x$ ) variations of the Péclet number for the same physical parameters as before, excluding $\varepsilon_{\mathrm{ev}}$, and for $M_{w}=100,000 \mathrm{Da}$, where the vertical dashed green line describes the separation where the transitions in the motion of the contact line and in the modus of deposition occur, the black and red points are given at the separations where the rate of evaporation, $\epsilon_{e v}$, was measured, and the dashed black and red lines give the trend of data.

more general, in the transition region between deposition of continuous states (Figs. 4(a,c)) and of non-continuous patterns (Figs. 4(f) to (i)). As the contact line moves away from the open end of the chamber we observe in both cases in Figs. 4(d,e) a rather sharp transition from the deposition of stripes to the deposition of a continuous film. This can be explained 
as caused by the continuous drift in the Péclet number. As indicated in Fig. 6(a), the Péclet number decreases with the increase of the distance between the open end of the chamber and the moving contact line, $x$, due to the decrease in the evaporation rate of the solvent. The latter results from the continuously increasing path length that the toluene vapor has to travel from the meniscus to the ambient air outside the chamber. A careful choice of experimental parameters allows us to commence our measurement with a large Péclet number at small $x$ and end the experiment with a much smaller Péclet number at large $x$ values (Fig. 6). The difference can be up to approximately two orders of magnitudes. This implies that the pattern can "drift" in the course of a single experimental procedure, changing from an non-continuous pattern deposition, found for large Péclet numbers, to continuous deposition, found for low Péclet numbers; see Figs. 4(d) and (e).

Finally, we conclude this part of the discussion by noting that although, overall we find some good qualitative agreement between the modeling results of the study by Fraštia and Thiele ${ }^{27}$ and our experimental results, there are also some points that call for cautiousness and require future improvements in model development and analysis:

(i) Even the most refined models are still rather restricted in the physical effects they allow for. Features that should be incorporated into the hydrodynamic models are the dependence of wettability on solute concentration, a distinction of dissolved solute and deposited solute (similar to the work conducted by Okuzono et al. ${ }^{66}$ ) and the possibility of phase-transitions of the solute-solvent mixture (as in ${ }^{67}$ ). All such additions need to be incorporated with care as potential cross-couplings can be easily missed. Recently, a general theoretical framework has been proposed for thin-films of complex fluids based on a gradient dynamics approach ( $\operatorname{se}^{\sqrt{6869}}$ and references therein) - the general approach has not yet been systematically applied to models for deposition from solution although several have been brought into the corresponding gradient form. ${ }^{70}$

(ii) The second issue concerns the analysis of the experimental patterns. Here we have determined pattern types and discussed their main features but have not given any further 
quantitative measures. In contrast, some theoretical works, based on time simulations, have analyzed the dependency of properties of line patterns on control parameters. ${ }^{7172} \mathrm{In}$ particular, the onset of pattern formation is of interest. Hypothesis regarding the underlying bifurcations are formulated elswhere ${ }^{\sqrt{27}}$ based on time simulations, while a different study ${ }^{\sqrt{73}}$ was able to employ continuation techniques to determine the full bifurcation diagram for line deposition in Langmuir-Blodgett transfer. Here, we see much future scope for detailed experimental analyses.

(iii) The final point concerns a major challenge faced by the theoretical approach. Nearly all mentioned theoretical studies of deposition patterns (and dip coating and LangmuirBlodgett transfer patterns) are limited to a one-dimensional (1d) substrate implying that the models can only distinguish between regularly spaced and irregularly spaced deposits of straight lines, and do not provide detailed information on transitions between the different two-dimensional (2d) pattern variants described here. There are a few exceptions in studies of the evaporative dewetting of nanoparticle suspensions ${ }^{\sqrt{60174}}$, Langmuir-Blodgett transfer of surfactant layers ${ }^{\sqrt{7017175}}$, and the dip-coating of a substrate in solutions ${ }^{\frac{70176}{7}}$ that do, however, not provide an overall picture in terms of a bifurcation analysis.

\section{The oscillatory wetting-dewetting motion}

Most of the previous experimental and theoretical studies in the literature describe the motion of the contact line during the process of pattern deposition as a stick-slip dynamics or as consisting of a sequence of pinning and depinning processes, which are connected to capillary forces, $\underset{77778}{ }$ the geometry of the deposits, ${ }^{[79}$ or viscous forces. ${ }^{[27}$ Here, however, we observe a different type of contact line dynamics, namely, cycles of oscillatory wettingdewetting motions of the contact line (Fig. 3). In each cycle the displacement during the dewetting phase is larger than the one in the wetting phase such that, overall, the liquid front recedes in time. The oscillatory motion of the contact line is likely the result of an additional effect, which is related to variations in the surface tension of the solution near the 
contact line. The variations in the surface tension appear to result as a consequence of a concentration-dependence of the surface tension, i.e., a Marangoni effect. Such effects were discussed previously ${ }^{80}$ but not in the context of a non-monotonic contact line motion, which is observed here.

In the case that the polymer possesses a lower surface tension than the solvent (in contact with air), the rapid increase in the concentration of the polymer near the contact line results in a decrease of the local surface tension of the solution close to the contact line. The resulting Marangoni forces are directed away from the contact line into the meniscus and cause a flow that pushes liquid from the vicinity of the contact line into the meniscus. 26181 In the opposite case, if the polymer possesses a higher surface tension than the solvent, a fast increase in the polymer concentration near the contact line causes a sharp local increase in the surface tension of the solution. The resulting Marangoni flow then pushes liquid from the bulk of the meniscus into the vicinity of the contact line. If the resulting flux is fast, the direction of contact line motion may reverse, resulting in the wetting phase of the cycle..$^{28 \mid 81 / 82}$ In our case, the surface tension of toluene at $20^{\circ} \mathrm{C}$ is approximately $28.4 \mathrm{mN} / \mathrm{m}$, while the surface tension of PMMA at the same conditions is approximately $40 \mathrm{mN} / \mathrm{m} .65$ Hence, one may expect that the accumulation of PMMA near the contact line increases the local surface tension and may trigger the observed phase of dynamic wetting.

To verify that the oscillatory motion of the contact line is not specific to the microchamber geometry, we also conduct a similar experiment employing a drop of a volatile polymer solution. The drop on a silicon oxide substrate, is placed at the bottom of a void composed of a glass box whose upper side has an adjustable opening as illustrated in Fig. 7. By adjusting the width of the opening, the contact area to the ambient air is controlled. This allows us to qualitatively alter the rate of evaporation of the drop. With this setup we also find the described oscillatory wetting-dewetting motion of the contact line, as illustrated by the snapshots in Fig. 8 and by the movie SI3 in the Supporting Information. Decreasing the evaporation rate by decreasing the size of the opening, we observe a transition in the contact 
line motion from stick-slip motion to oscillatory wetting-dewetting motion. The sequence of frames in the figure shows two cycles of the latter with the contact line marked by the red arrow. Following the deposition of the stripe (shown in (a) as a light diffraction pattern), which is placed parallel to and on the right side of the contact line, the contact line recedes and dewets the substrate until its motion is arrested in (b). Then, the contact line moves again but in the opposite direction wetting the substrate again, as shown in (c). Images (d) to (f) present the next dewetting-wetting cycle, where the deposition of a new stripe takes place in (d). The solution dewets the substrate until being arrested again in (e). Then, the motion of the contact line again changes direction and the solution wets the substrate. The next stripe deposition occurs at the position of the contact line in (f).

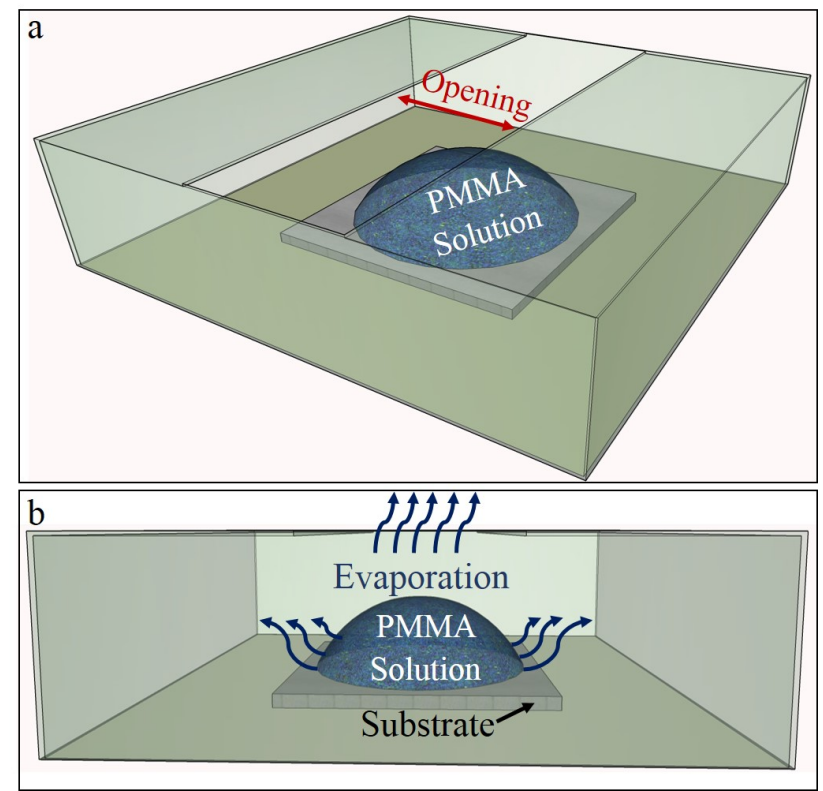

Figure 7: Sketches of the (a) top view and (b) side view of the semi-closed void system used to qualitatively control the rate of evaporation for a sessile drop of a volatile polymer solution. 

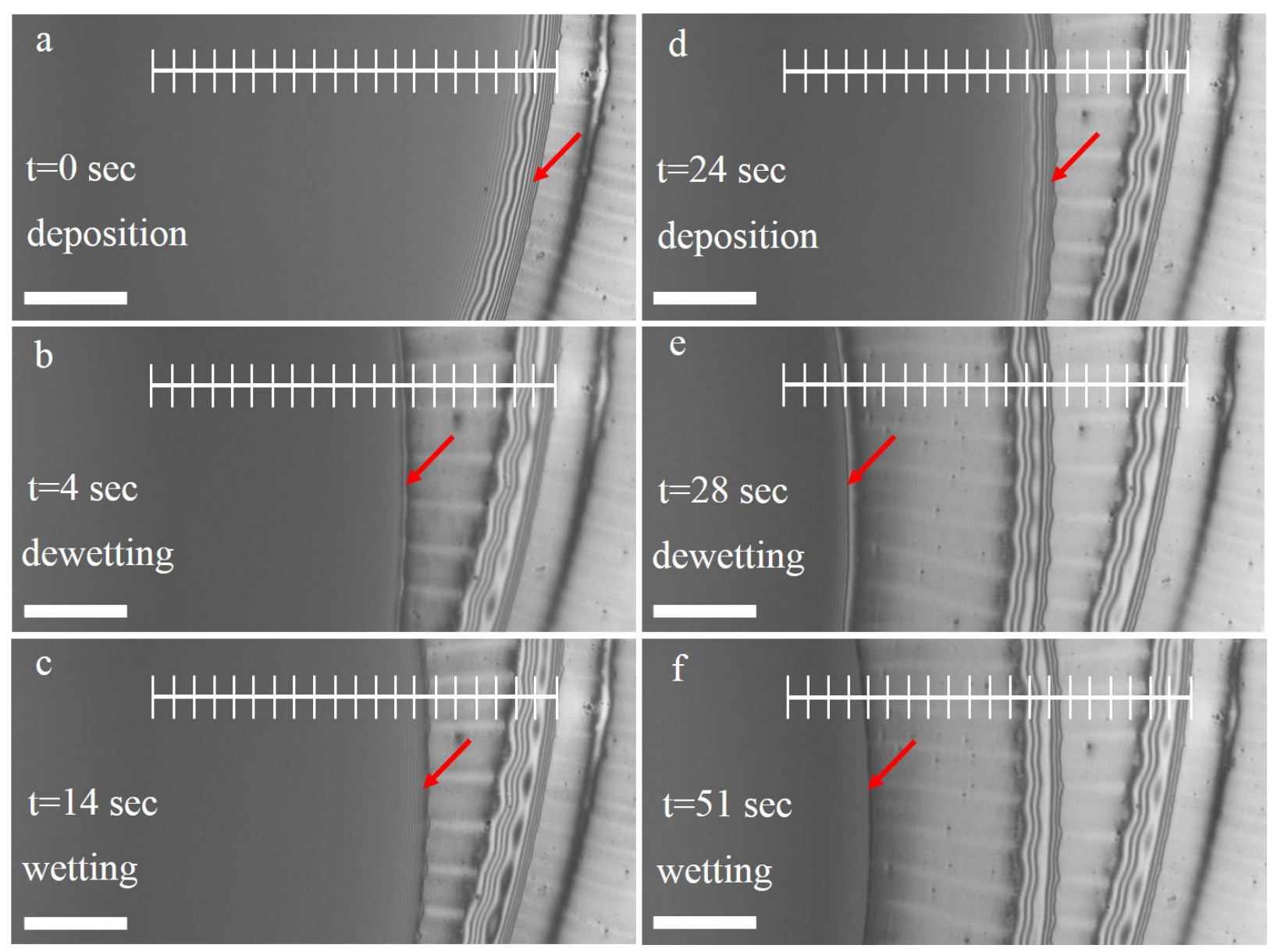

Figure 8: The oscillatory wetting-dewetting motion of the three phase contact line of a drop inside a cavity, which is partially open to the atmosphere, is depicted as a sequence of optical microscope frames. Frames (a-c) and (d-f) respectively represent two subsequent cycles. The contact line (marked by a red arrow) separates the solution on the left (appearing as a nearly uniform patch of gray) and the polymer deposit on the right. Both, the polymer deposit and the liquid in the vicinity of the contact line appear as dark and bright fringes due to the diffraction of light. The temperature is $T=25^{\circ} \mathrm{C}$, the initial polymer (PMMA) concentration is $C=1 \mathrm{mg} / \mathrm{ml}$, and $M_{w}=350,000$ Da. The scale bars shows $100 \mu \mathrm{m}$ and the tick separation is $20 \mu \mathrm{m}$.

\section{Conclusions}

We have studied the evaporative dewetting of a volatile polymer solution and the patterns of polymeric deposit left behind by the moving three-phase contact line. Two geometries, namely, a meniscus in a micro-chamber and a sessile drop in a semi-closed void have consis- 
tently shown the occurrence of different modi of contact line motion and deposition depending on initial polymer concentration, molecular mass of the polymer, and ambient temperature.

We have shown that the motion of the meniscus is governed by a Péclet number, characterizing the ratio between the convection of the solution and the diffusion of the solvent. It is also proportional to the rate of liquid evaporation. A small Péclet number results in diffusion-dominated transport of polymer chains, which causes a nearly homogeneous solute concentration in the solution and a monotonic contact line motion. This in turn gives either a continuous deposition of a nearly uniform polymer film or a continuous deposition that does not dry as a uniform solid polymer film but undergoes a further continuous dewetting step, resulting in a dry polymer film which contains a isotropic arrangement of many small holes or a random continuous polygonal network.

In contrast, at large Péclet numbers, polymer diffusion is very slow as compared to the evaporation-induced convective flux. Ultimately, this convection-dominated transport results in the deposition of various types of non-continuous patterns, namely, regular sequences of parallel lines, line patterns with side protrusions caused by transversal contact line instabilities, and "punch-hole-like" patterns caused by strong transversal contact line instabilities. Using morphological phase diagrams we have summarized how the type of deposit depends on temperature and initial concentration. Notably, in this case the contact line may show either the usual stick-slip motion where in the slip phase the contact line recedes, i.e., dewetting of the solid, or an oscillatory wetting-dewetting motion where the contact line ratchets back and forth, on average resulting in a receding motion. Both motion types support deposits in the form of stripes, and further may show fingering instabilities during the dewetting phase when increasing the temperature and/or concentration in the cases of higher molecular weight.

We have explained the mechanisms responsible for the oscillatory wetting-dewetting motion as follows: The evaporation of the solvent supports the accumulation of the polymeric solute close to the contact line. This increase in solute concentration increases the local 
surface tension and the resulting Marangoni flow is directed towards the contact line and supports the dynamic wetting phase. Eventually, the continuously ongoing evaporation of the solvent solidifies (diverging viscosity at gelling/jamming transition) the solution near the contact line to a level where the motion stops. Then the solution away from the contact line breaks from the solidified part and undergoes a fast convective dewetting before solvent evaporation dominates again. When the concentration of the solute near the contact line is again sufficiently increased to alter the direction of motion of the contact line, again the dynamic wetting phase occurs and the cycle starts from the beginning.

A qualitative control over the rate of solvent evaporation from a drop of the same polymer solution in a void has further shown that the wetting-dewetting motion cycles are not limited to the micro-chamber geometry but occur in different confined geometries. In both considered geometries one may shift from a stick-slip motion of the contact line to an oscillatory wettingdewetting motion by altering the evaporation rate.

We have compared our experimental results to theoretical results, which are available in the literature. The theory predicts the deposition of periodic lines in several similar settings. We find qualitative agreement in several major points. Most important of which is the connection between the morphology of the deposit and the ratio between the rate of convection and diffusion of the polymer chains in the volatile solvent. However, we have also

pointed out in which way the existing models are limited and explained which additional effects should be included in future theoretical models.

\section{Acknowledgements}

We acknowledge support of this research by the German Israel Foundation for Scientific Research and Development (GIF) under grant number I-1361-401.10/2016. 


\section{References}

(1) Deegan, R. D.; Bakajin, O.; Dupont, T. F.; Huber, G.; Nagel, S. R.; Witten, T. A. Capillary flow as the cause of ring stains from dried liquid drops. Nature 1997, 389, $827-829$.

(2) Deegan, R. D. Pattern formation in drying drops. Phys. Rev. E 2000, 61, 475.

(3) Thiele, U. Entnetzung von Kollagenfilmen. Ph.D. thesis, Technische Universität Dresden, Dresden, 1998.

(4) Pauliac-Vaujour, E.; Stannard, A.; Martin, C.; Blunt, M. O.; Notingher, I.; Moriarty, P.; Vancea, I.; Thiele, U. Fingering instabilities in dewetting nanofluids. Phys. Rev. Lett 2008, 100, 176102.

(5) Lin, Y.; Su, Z.; Balizan, E.; Niu, Z.; Wang, Q. Controlled Assembly of Protein in Glass Capillary. Langmuir 2010, 26, 12803-12809.

(6) Mhatre, S.; Zigelman, A.; Abezgauz, L.; Manor, O. Influence of a Propagating Megahertz Surface Acoustic Wave on the Pattern Deposition of Solute Mass off an Evaporating Solution. Langmuir 2016, 32, 9611-9618.

(7) Byun, M.; Bowden, N. B.; Lin, Z. Hierarchically Organized Structures Engineered from Controlled Evaporative Self-Assembly. Nano Lett. 2010, 10, 3111-3117.

(8) Xu, J.; Xia, J.; Hong, S.; Lin, Z.; Qiu, F.; Yang, Y. Self-assembly of gradient concentric rings via solvent evaporation from a capillary bridge. Phys. Rev. Lett. 2006, 96, 066104.

(9) Weissman, J.; Sunkara, H.; Tse, A.; Asher, S. Thermally switchable periodicities and diffraction from mesoscopically ordered materials. Science. 1996, 274, 959.

(10) Xia, Y.; Gates, B.; Yin, Y.; Lu, Y. Monodispersed colloidal spheres: old materials with new applications. Adv. Mater. 2000, 12, 693-713. 
(11) Homede, E.; Abo Jabal, M.; Ionescu, R.; Haick, H. Printing Ultrasensitive Artificially Intelligent Sensors Array with a Single-Self Propelled Droplet Containing Nanoparticles. Adv. Funct. Mater. 2016, 26, 6359-6370.

(12) Asher, S.; Alexeev, V.; Goponenko, A.; Sharma, A.; Lednev, I.; Wilcox, C.; Finegold, D. Photonic crystal carbohydrate sensors: low ionic strength sugar sensing. JACS 2003, 26, 3322-3329.

(13) Velev, O.; Kaler, E. In situ assembly of colloidal particles into miniaturized biosensors. Langmuir 1999, 15, 3693-3698.

(14) Lu, Y.; Liu, G.; Kim, J.; Mejia, Y.; Lee, L. Nanophotonic crescent moon structures with sharp edge for ultrasensitive biomolecular detection by local electromagnetic field enhancement effect. Nano Lett. 2005, 5, 119-124.

(15) Sun, S.; Murray, C.; Weller, D.; Folks, L.; Moser, Monodisperse FePt nanoparticles and ferromagnetic FePt nanocrystal superlattices. Science 2000, 287, 1989-1992.

(16) Pham, H.; Gourevich, I.; Oh, J.; Jonkman, J.; Kumacheva, E. A multi dye nanostructured material for optical data storage and security data encryption. Advanced Materials. Adv. Mater. 2004, 16, 516-520.

(17) Joannopoulos, J.; Villeneuve, P.; Fan, S. Photonic crystals: putting a new twist on light. Nature 1997, 386, 143.

(18) Tetreault, N.; Míguez, H.; Ozin, G. Silicon inverse opal-a platform for photonic bandgap research. Adv. Mater. 2004, 16, 1471-1476.

(19) Aizpurua, J.; Hanarp, P.; Sutherland, D.; Käll, M.; Bryant, G.; De Abajo, F. Optical properties of gold nanorings. Phys. Rev. Lett. 2003, 90, 057401.

(20) Sun, C.; Linn, N.; Jiang, P. Templated fabrication of periodic metallic nanopyramid arrays. Chem. Mater. 2007, 19, 4551-4556. 
(21) Velev, O.; Jede, T.; Lobo, R.; Lenhoff, A. Porous silica via colloidal crystallization. Nature 1997, 389, 447.

(22) Holland, B.; Blanford, C.; Stein, A. Synthesis of macroporous minerals with highly ordered three-dimensional arrays of spheroidal voids. Science 1998, 281, 538-540.

(23) Park, S.; Xia, Y. Macroporous Membranes with Highly Ordered and ThreeDimensionally Interconnected Spherical Pores. Adv. Mater. 1998, 10, 1045-1048.

(24) Braun, P.; Wiltzius, P. Microporous materials: Electrochemically grown photonic crystals. Nature 1999, 402, 603-604.

(25) Hu, H.; Larson, R. Marangoni effect reverses coffee-ring depositions. J. Phys. Chem. B 2006, 110, 7090-7094.

(26) Abo Jabal, M.; Homede, E.; Pismen, L.; Haick, H.; Leshansky, A. Controlling Marangoni flow directionality: patterning nano-materials using sessile and sliding volatile droplets. Eur. Phys. J. Spec. Top. 2017, 226, 1307-1324.

(27) Fraštia, A., L.and Archer; Thiele, U. Modelling the formation of structured deposits at receding contact lines of evaporating solutions and suspensions. Soft Matter 2012, 8, $11363-1138$.

(28) Fanton, X.; Cazabat, A. Spreading and instabilities induced by a solutal Marangoni effect. Langmuir 1998, 14, 2554-2561.

(29) Bodiguel, H.; Doumenc, F.; Guerrier, B. Stick-slip patterning at low capillary numbers for an evaporating colloidal suspension. Langmuir 2010, 26, 10758-10763.

(30) Thiele, U. Patterned deposition at moving contact lines. Adv. Colloid. Interface. Sci. 2014, 206, 399-413.

(31) Weon, B.; Je, J. Self-pinning by colloids confined at a contact line. Phys. Rev. Lett. 2013, 110, 028303. 
(32) Maheshwari, S.; Zhang, L.; Zhu, Y.; Chang, H. Coupling between precipitation and contact-line dynamics: Multiring stains and stick-slip motion. Physical review letters. Phys. Rev. Lett. 2008, 100, 044503.

(33) Kajiya, T.; Nishitani, E.; Yamaue, T.; Doi, M. Piling-to-buckling transition in the drying process of polymer solution drop on substrate having a large contact angle. Phys. Rev. E 2006, 73, 011601.

(34) Gorand, Y.; Pauchard, L.; Calligari, G.; Hulin, J.; Allain, C. Mechanical instability induced by the desiccation of sessile drops. Langmuir 2004, 20, 5138-5140.

(35) Han, W.; Lin, Z. Learning from "Coffee Rings": Ordered Structures Enabled by Controlled Evaporative Self-Assembly. Angew. Chem. Int. Ed. 2012, 51, 1534-1546.

(36) Byun, M.; Han, W.; Qiu, F.; Bowden, N.; Lin, Z. Hierarchically Ordered Structures Enabled by Controlled Evaporative Self-Assembly. Small 2010, 6, 2250-2255.

(37) Hong, S.; Xia, J.; Lin, Z. Spontaneous formation of mesoscale polymer patterns in an evaporating bound solution. Adv. Mater. 2007, 19, 1413-1417.

(38) Xu, J.; Xia, J.; Lin, Z. Evaporation-Induced Self-Assembly of Nanoparticles from a Sphere-on-Flat Geometry. Angew. Chem. 2007, 119, 1892-1895.

(39) Denkov, N.; Velev, O.; Kralchevski, P.; Ivanov, I.; Yoshimura, H.; Nagayama, K. Mechanism of formation of two-dimensional crystals from latex particles on substrates. Langmuir 1992, 8, 3183-3190.

(40) Pauliac, V. E.; Moriarty, P. Meniscus-mediated organization of colloidal nanoparticles. J. Phys. Chem. C 2007, 111, 16255-16260.

(41) Kwon, S.; Yoon, D.; Yang, W. A simple route of ordered high quality mesoscale stripe polymer patterns. Soft Matter 2011, 7, 1682-1685. 
(42) Leng, J. Drying of a colloidal suspension in confined geometry. Phys Rev E 2010, 82, 021405 .

(43) Li, X.; Wang, C.; Shao, J.; Ding, Y.; Tian, H.; Li, X.; Wang, L. Periodic Parallel Array of Nanopillars and Nanoholes Resulting from Colloidal Stripes Patterned by Geometrically Confined Evaporative Self-Assembly for Unique Anisotropic Wetting. Appl. Mater. Interfaces 2014, 6, 20300-20308.

(44) Zeng, H.; Kristiansen, K.; Wang, P.; Bergli, J.; Israelachvili, J. Surface-induced patterns from evaporating droplets of aqueous carbon nanotube dispersions. Langmuir 2011, 27 , $7163-7167$.

(45) Abkarian, M.; Nunes, J.; Stone, H. Colloidal crystallization and banding in a cylindrical geometry. J. Am. Chem. Soc. 2004, 126, 5978-5979.

(46) Larson, R. Transport and deposition patterns in drying sessile droplets. AIChE J 2014, $60,1538-1571$.

(47) Yabu, H.; Shimomura, M. Preparation of Self Organized Mesoscale Polymer Patterns on a Solid Substrate: Continuous Pattern Formation from a Receding Meniscus. Adv. Funct. Mater. 2005, 15, 575-581.

(48) Hong, S.; Xia, J.; Byun, M.; Zou, Q.; Lin, Z. Mesoscale patterns formed by evaporation of a polymer solution in the proximity of a sphere on a smooth substrate: molecular weight and curvature effects. Macromolecules 2007, 40, 2831-2836.

(49) Nguyen, V.; Stebe, K. Patterning of small particles by a surfactant-enhanced Marangoni-Bénard instability. Phys. Rev. Lett. 2002, 88, 164501.

(50) Maillard, M.; Motte, L.; Pileni, M. Rings and hexagons made of nanocrystals. Adv. Mater. 2001, 13, 200-204. 
(51) Zigelman, A.; Manor, O. A model for pattern deposition from an evaporating solution subject to contact angle hysteresis and finite solubility. Soft Matter 2016, 12, 56935707.

(52) Masaro, L.; Zhu, X. Physical models of diffusion for polymer solutions, gels and solids. Prog. Polym. Sci. 1999, 24, 731-775.

(53) Mertig, M.; Thiele, U.; Bradt, J.; Leibiger, G.; Pompe, W.; Wendrock, H. Scanning Force Microscopy and Geometrical Analysis of Two-DimensionalCollagen Network Formation. Surface and Interface Analysis 1997, 25, 514-521.

(54) Thiele, U.; Mertig, M.; Pompe, W. Dewetting of an evaporating thin liquid film: Heterogeneous nucleation and surface instability. Phys. Rev. Lett. 1998, 80, 2869-2872.

(55) Thiele, U.; Velarde, M. G.; Neuffer, K. Dewetting: Film rupture by nucleation in the spinodal regime. Phys. Rev. Lett. 2001, 87, 016104.

(56) Thiele, U. Open questions and promising new fields in dewetting. Eur. Phys. J. E 2003, 12, 409-416.

(57) Thiele, U.; Mertig, M.; Pompe, W.; Wendrock, H. Foams and Emulsions; Kluver Academic Publishers, 1999; pp 127-136, Proceedings of the NATO advanced study institute on foams, emulsions and cellular materials, Cargèse Corsica 5/97.

(58) Reiter, G. Unstable thin polymer films: Rupture and dewetting processes. Langmuir 1993, 9, 1344-1351.

(59) Moriarty, P.; Taylor, M. D. R.; Brust, M. Nanostructured cellular networks. Phys. Rev. Lett. 2002, 89, 248303.

(60) Robbins, M. J.; Archer, A. J.; Thiele, U. Modelling the evaporation of thin films of colloidal suspensions using Dynamical Density Functional Theory. J. Phys.: Condens. Matter 2011, 23, 415102. 
(61) Frastia, L.; Archer, A. J.; Thiele, U. Dynamical model for the formation of patterned deposits at receding contact lines. Phys. Rev. Lett. 2011, 106, 077801.

(62) Thiele, U.; Vancea, I.; Archer, A. J.; Robbins, M. J.; Frastia, L.; Stannard, A.; PauliacVaujour, E.; Martin, C. P.; Blunt, M. O.; Moriarty, P. J. Modelling approaches to the dewetting of evaporating thin films of nanoparticle suspensions. J. Phys.-Cond. Mat. 2009, 21, 264016.

(63) Oron, A.; Davis, S.; Bankoff, S. Long-scale evolution of thin liquid films. Rev. Mod. Phys. 1997, 69, 931.

(64) Warner, M.; Craster, R.; Matar, O. Surface patterning via evaporation of ultrathin films containing nanoparticles. J. Colloid Interface Sci. 2003, 267, 92-110.

(65) Brandrup, I. E., J.; McDowell, W. Polymer handbook; Wily, 1975.

(66) Okuzono, T.; Kobayashi, M.; Doi, M. Final shape of a drying thin film. Phys. Rev. E 2009, 80, 021603.

(67) Náraigh, L. Ó.; Thiffeault, J. L. Nonlinear dynamics of phase separation in thin films. Nonlinearity 2010, 23, 1559-1583.

(68) Thiele, U.; Archer, A.; Pismen, L. Gradient dynamics models for liquid films with soluble surfactant. Phys. Rev. Fluids 2016, 1, 083903.

(69) Thiele, U. Recent advances in and future challenges for mesoscopic hydrodynamic modelling of complex wetting. Colloids Surf. A 2018, 553, 487-495.

(70) Wilczek, M.; Tewes, W. B. H.; Gurevich, S. V.; Köpf, M. H.; Chi, L.; Thiele, U. Modelling Pattern Formation in Dip-Coating Experiments. Math. Model. Nat. Phenom. 2015, 10, 44-60.

(71) Köpf, M. H.; Gurevich, S. V.; Friedrich, R.; Thiele, U. Substrate-mediated pattern formation in monolayer transfer: a reduced model. New J. Phys. 2012, 14, 023016. 
(72) Dey, M.; Doumenc, F.; Guerrier, B. Numerical simulation of dip-coating in the evaporative regime. Eur. Phys. J. E 2016, 39, 19.

(73) Köpf, M. H.; Thiele, U. Emergence of the bifurcation structure of a Langmuir-Blodgett transfer model. Nonlinearity 2014, 27, 2711-2734.

(74) Vancea, I.; Thiele, U.; Pauliac-Vaujour, E.; Stannard, A.; Martin, C. P.; Blunt, M. O.; Moriarty, P. J. Front instabilities in evaporatively dewetting nanofluids. Phys. Rev. E 2008, 78, 041601.

(75) Li, L.; Köpf, M.; Gurevich, S.; Friedrich, R.; Chi, L. Structure Formation by Dynamic Self-Assembly. Small 2012, 8, 488-503.

(76) Engelnkemper, S.; Gurevich, S.; Uecker, H.; Wetzel, D.; Thiele, U. In Computational Modeling of Bifurcations and Instabilities in Fluid Mechanics; Gelfgat, A., Ed.; Springer, 2018; Chapter Continuation for thin film hydrodynamics and related scalar problems, (at press).

(77) Thompson, P.; Robbins, M. Simulations of contact-line motion: slip and the dynamic contact angle. Phys. Rev. Lett. 1989, 63, 766.

(78) Hoffman, R. A study of the advancing interface. I. Interface shape in liquid-gas systems. J. Colloid Interface Sci. 1975, 50, 228-241.

(79) Bodiguel, H.; Doumenc, F.; Guerrier, B. Stick-Slip patterning at low capillary numbers for an evaporating colloidal suspension. Langmuir 2010, 26, 10758-10763.

(80) Doumenc, F.; Guerrier, B. Self-patterning induced by a solutal Marangoni effect in a receding drying meniscus. Europhysics Letters (EPL) 2013, 103, 14001.

(81) Poulard, C.; Damman, P. Control of spreading and drying of a polymer solution from Marangoni flows. EPL 2007, 80, 64001. 
(82) Pesach, D.; Marmur, A. Marangoni effects in the spreading of liquid mixtures on a solid. Langmuir 1987, 3, 519-524. 